\title{
Experimental tests of Chiral Perturbation Theory
}

\author{
H. Merkel ${ }^{\mathrm{a}}$ \\ Institut für Kernphysik, Johannes Gutenberg-Universität Mainz, D-55099 Mainz, Germany \\ / \\ Published online: 15 May 2006 - C Società Italiana di Fisica / Springer-Verlag 2006
}

\begin{abstract}
Over the last decade, a series of dedicated experiments to test heavy baryon chiral perturbation theory was performed at MAMI. Photo production of neutral pions close to threshold with unpolarized and polarized photon beam was performed to separate the multipole amplitudes at threshold. The extension of this experiments to a modest photon virtuality of $Q^{2}<0.1 \mathrm{GeV}^{2} / c^{2}$ was performed to extract additional longitudinal multipoles and to exploit the $Q^{2}$ evolution predicted by theory. An out-of-plane measurement above $\pi^{+}$threshold with polarized electron beam gave access to the imaginary part of the $s$-wave amplitude. Finally, by coherent photo and electro production from the deuteron the neutron amplitude could be extracted.
\end{abstract}

PACS. 25.30.Rw Electroproduction reactions - 13.60.Le Meson production - 12.39.Fe Chiral Lagrangians

\section{Introduction}

Chiral perturbation theory is an effective field theory, which utilizes the symmetry properties of the QCD Lagrangian to extract observables at low momenta in a systematic fashion (see, e.g., S. Scherer, this issue).

Meanwhile, a vast amount of observables can be calculated in the framework of chiral perturbation theory, leading to numerous ways to test this theory in experiment. This contribution, however, concentrates on a dedicated series of experiments, which were especially designed to test the predictions of Heavy-Baryon Chiral Perturbation Theory (HBChPT).

The investigated reaction is the electromagnetic production of the Goldstone Boson of the theory, the pion. The choice of the electromagnetic probe ensures the precision, which is necessary to extract amplitudes very close to threshold. While the $\pi^{+}$production is dominated by the charge (Kroll-Ruderman term), the $\pi^{0}$ production is the ideal testing ground for HBChPT.

Over the last decade, several experiments on $\pi^{0}$ threshold production were performed at MAMI and at other electron accelerator laboratories. The formalism of HBChPT was developed in parallel with large success in predicting or fitting of the measured threshold observables, leading to an improving consistent picture. In this article, an overview over the existing experiments and their interpretation in terms of $\mathrm{HBChPT}$ is given.

\footnotetext{
a e-mail: merkel@kph.uni-mainz.de
}

\section{Neutral pion photo production}

Since chiral perturbation theory is an expansion in small momenta, only for a small region above threshold predictions can be derived. Close to threshold, the angular structure of the differential cross section of photo production of pseudoscalar mesons can be expanded in

$$
\sigma(\theta)=\frac{q}{k}\left(A+B \cdot \cos \theta+C \cdot \cos ^{2} \theta\right),
$$

with $q$ and $k$ the pion and photon center of mass momentum and $\theta$ the pion center of mass production angle.

The angular coefficients can be further decomposed in $s$ - and $p$-wave multipoles:

$$
\begin{aligned}
& A=E_{0+}^{2}+\frac{1}{2}\left(P_{2}^{2}+P_{3}^{2}\right), \\
& B=2 \cdot \operatorname{Re}\left(E_{0+} P_{1}{ }^{*}\right), \\
& C=P_{1}^{2}-\frac{1}{2}\left(P_{2}^{2}+P_{3}^{2}\right) .
\end{aligned}
$$

In this field, it is common to use the $p$-wave combinations

$$
\begin{aligned}
& P_{1}=3 E_{1+}+M_{1+}-M_{1-}, \\
& P_{2}=3 E_{1+}-M_{1+}+M_{1-}, \\
& P_{3}=2 M_{1+}+M_{1-} .
\end{aligned}
$$

Not only the angular form of the multipoles is known, but also their energy dependence. The $p$-waves rise proportional to photon and pion CMS momentum $q \cdot k$, while the form of the $s$-wave multipole is dictated by unitary, as will be discussed later.

The first experiments on threshold $\pi^{0}$ photo production $[1,2]$ were designed to test the Low-Energy Theorem 


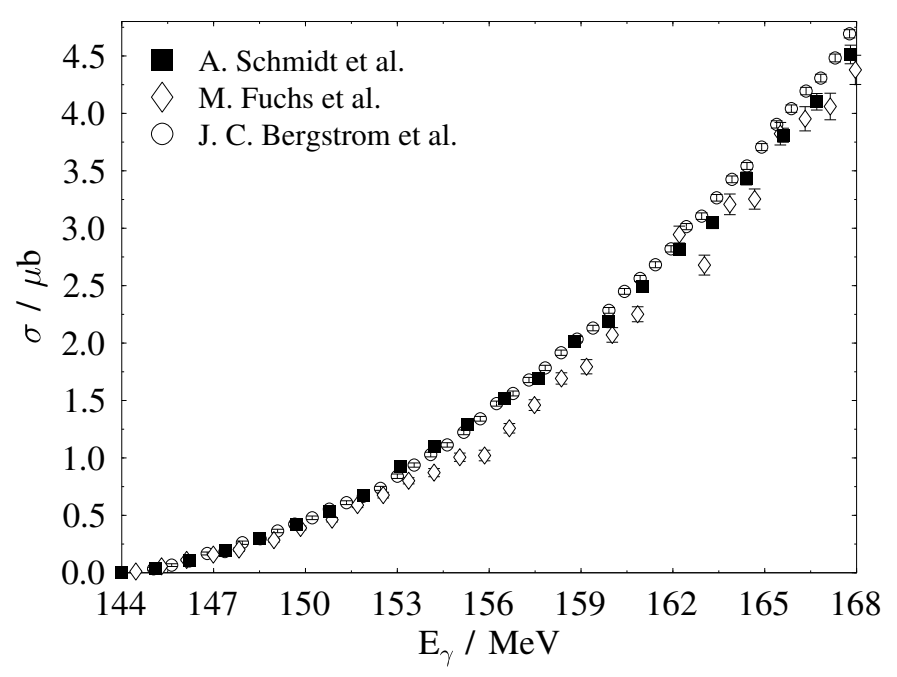

Fig. 1. Total cross section of neutral pion photo production (from [3]).
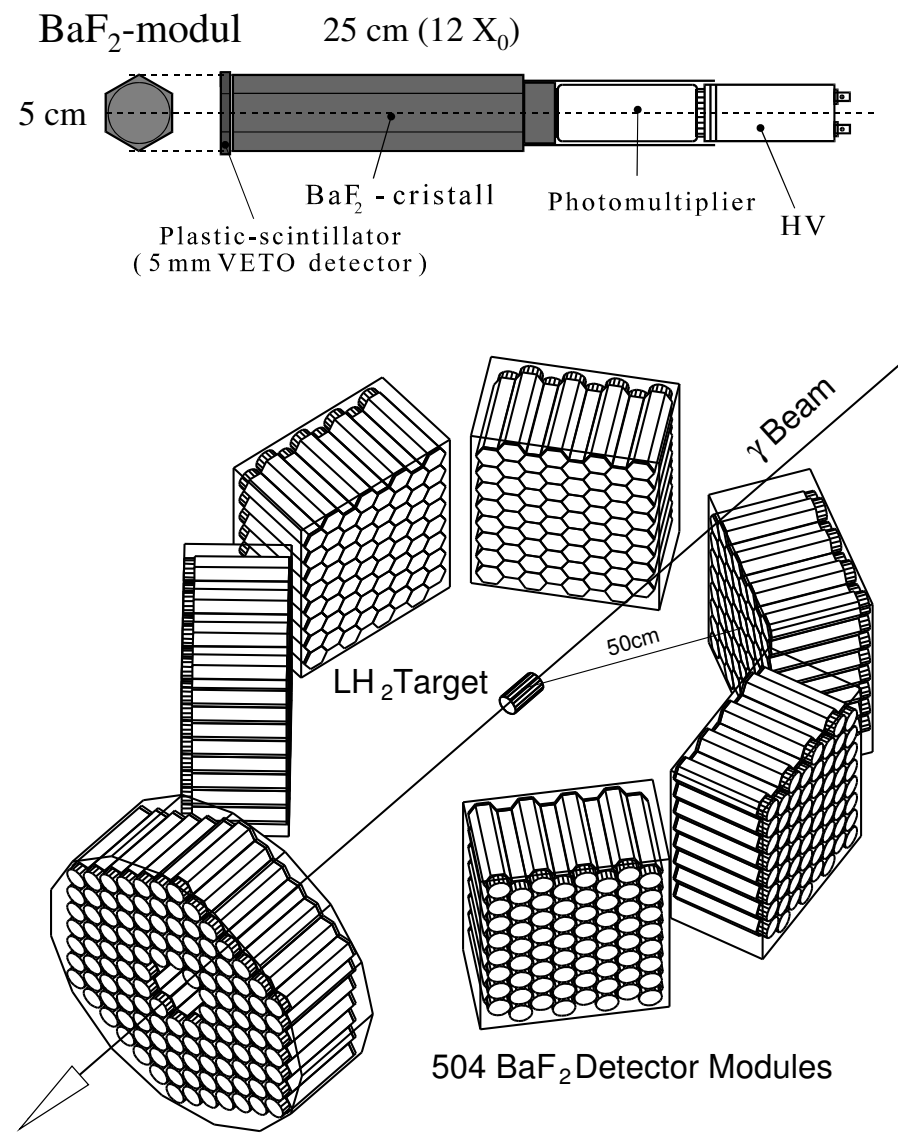

Fig. 2. One BaF module and the complete setup of the photon spectrometer TAPS at the MAMI A2-Collaboration.

(LET) [4], which derived from general principles a value for the $s$-wave amplitude $E_{0+}$ at threshold.

These first experiments showed a serious discrepancy from the prediction of the low-energy theorem. The development of the formalism of Heavy-Baryon Perturbation Theory resolved this puzzle by showing, that the LET value is only the leading term in a slow converging series [5]. These authors calculated $s$ - and $p$-waves and showed, that while $E_{0+}$ and $P_{3}$ are slow converging, the $P_{1}$ and $P_{2}$ multipoles were fast converging and should be a strong prediction of HBChPT.

Figure 1 shows the total cross section of the last MAMI experiment [3] in comparison with former data from SAL [6] and MAMI [7]. The data were taken at the tagged photon beam of the MAMI A2 Collaboration (fig. 2). The reaction was identified by the detection of the two decay photons of the pion in coincidence with the TAPS detector, an array of $504 \mathrm{BaF}$ detector modules arranged in 7 blocks.

With this setup, differential cross sections were measured up to an incoming photon laboratory energy of $168 \mathrm{MeV}$. By fitting the angular coefficients $A, B$, and $C$ to the differential cross section and extrapolating to threshold by using the known energy dependence, the multipole combinations Re $E_{0+}, P_{1}$, and $P_{2}^{2}+P_{3}^{2}$ could be extracted.

\subsection{Polarized photon asymmetry}

To further decompose $P_{2}^{2}+P_{3}^{2}$, an additional polarization observable has to be measured. The polarized photon asymmetry $\Sigma$ is defined as the asymmetry of the cross section in respect to the polarization plane of a polarized photon beam, i.e.

$$
\Sigma=\sigma_{0}\left(1-P_{\gamma} \Sigma(\theta) \cos 2 \phi\right)
$$

with $\phi$ the angle between polarization plane and production plane and $P_{\gamma}$ the photon polarization. This asymmetry is proportional to the difference $P_{2}^{2}-P_{3}^{2}$ and allows the decomposition of the modulus of these two multipole amplitudes.

At MAMI, a polarized photon beam was prepared by coherent Bremsstrahlung from a diamond crystal. The asymmetry was again determined by detecting the decay photons of the pion with the TAPS detector. Figure 3

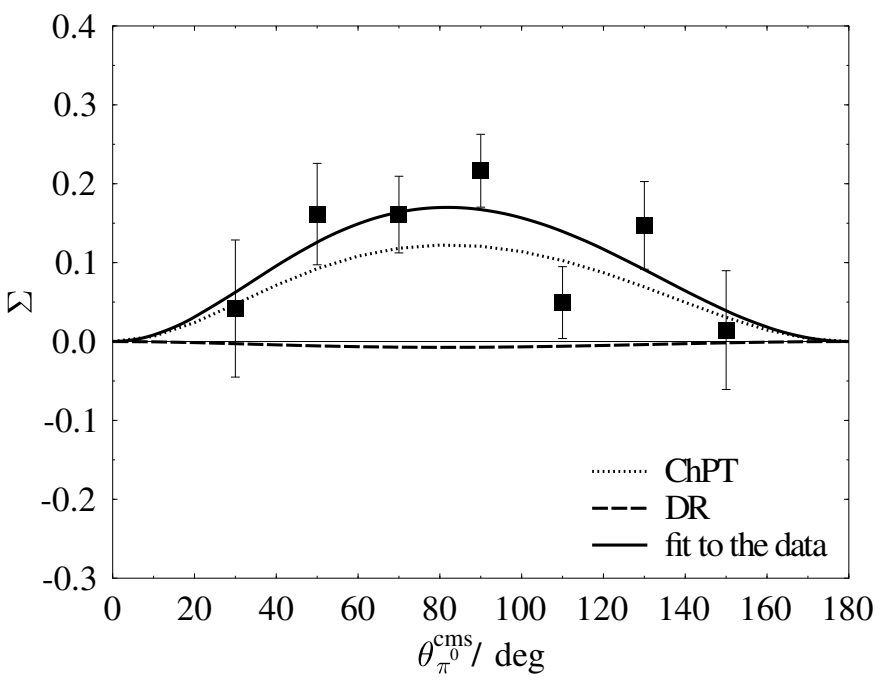

Fig. 3. The polarized photon asymmetry $\Sigma$ (from [3]). 
Table 1. Multipole amplitudes extracted in photoproduction.

\begin{tabular}{|c|c|c|c|c|}
\hline & $\begin{array}{c}E_{0+} \\
\left(10^{-3} / m_{\pi}\right)\end{array}$ & \multicolumn{3}{|c|}{$\left(q \cdot k \cdot 10^{-3} / m_{\pi}^{3}\right)$} \\
\hline MAMI & $-1.31 \pm 0.08$ & $10.02 \pm 0.2$ & $-10.5 \pm 0.2$ & $13.1 \pm 0.1$ \\
\hline $\mathrm{SAL}$ & $-1.32 \pm 0.05$ & $10.26 \pm 0.1$ & & \\
\hline ChPT & -1.16 & $10.33 \pm 0.6$ & $-11.0 \pm 0.6$ & $11.7 \pm 0.6$ \\
\hline DR & -1.22 & 10.54 & -11.4 & 10.2 \\
\hline
\end{tabular}

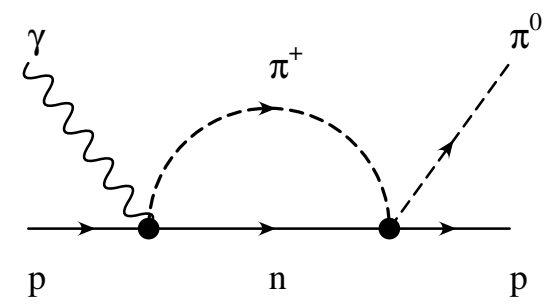

Fig. 4. Rescattering graph.

shows the measured asymmetry, averaged over the energy range.

Combining the unpolarized and polarized measurement, the four threshold amplitudes can be extracted. Table 1 summarizes these amplitudes. For $E_{0+}$ and $P_{1}$, the values can be compared with the data from an experiment at SAL [6], which are in agreement within the error bar. The quoted values of HBChPT, which are based on a refit of the low-energy constants [8] also successfully describe the data. For comparison, also the results of a dispersion relations calculation [9] is added. While the values for $P_{2}$ and $P_{3}$ are reasonable in the table, in fig. 3 the remaining small discrepancy is more pronounced due to the large sensitivity of $\Sigma$ to the difference of these two amplitudes.

\subsection{Cusp effect of the s-wave amplitude}

While the $p$-wave amplitudes rise proportional to the momenta $q \cdot k$ the energy dependence of the $s$-wave amplitude is given by the unitarity of the scattering matrix.

Chiral perturbation theory alone predicts a roughly constant $s$-wave amplitude. The $\pi^{0} p$ amplitude, however, is an order of magnitude smaller than the $\pi^{+} n$ amplitude due to the sizable Kroll-Ruderman term. Above the $\pi^{+} n$ threshold, a photon coupling to a $\pi^{+}$in the intermediate state with a following pion charge exchange in the final state (see fig. 4) leads to a large contribution to the $s$ wave amplitude.

Figure 5 shows the real part of the $E_{0+}$ amplitude in photo production extracted from the MAMI data [3]. The two different data sets are extracted from the same differential cross section by the two assumptions of the $p$-waves rising proportional to $q$ (open squares) or proportional to $q \cdot k$ (filled squared). The first assumption is phenomenological valid over a larger energy range, while the second assumption corresponds to the prediction of chiral perturbation theory.

The cusp effect at the $\pi^{+} n$ threshold is clearly seen Again, the dispersion relations calculation [9] is included

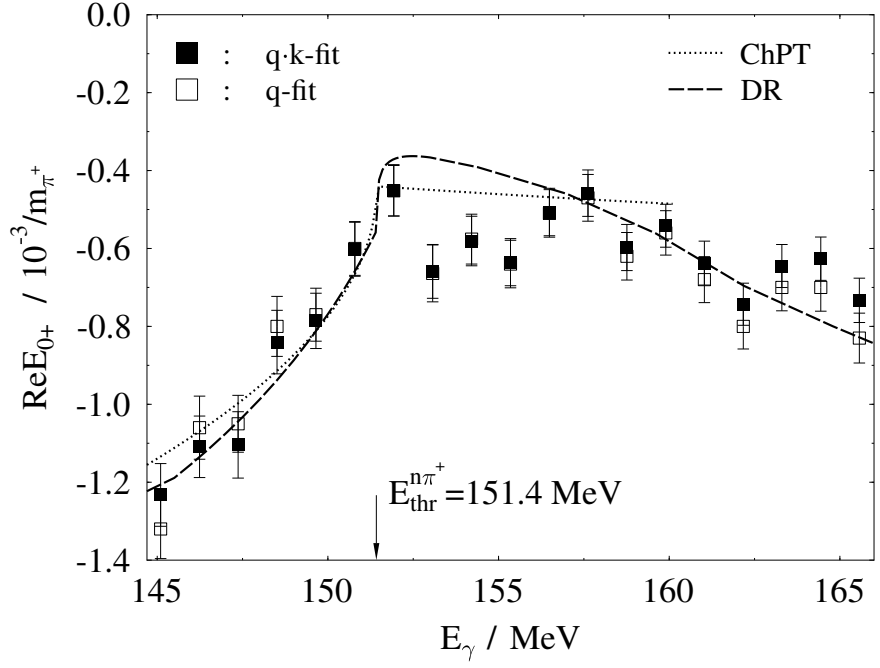

Fig. 5. Real part of the $s$-wave multipole $E_{0+}$ (see [3] for details).

(dashed line). The strength of this effect is roughly given by the $E_{0+}$ amplitude of the $\pi^{+} n$ production times the charge exchange scattering length.

Only the real part of $E_{0+}$ could be extracted by this experiment. For the imaginary part, an additional polarization experiment would be necessary, e.g., a measurement of the polarized target asymmetry.

\section{Electroproduction}

While in photo production two amplitudes are given by a fit of low-energy constants to the data, the extension to electro production exploits additional predictive power of HBChPT. In addition to the dependence of the multipoles on the photon virtuality $Q^{2}$ the two longitudinal $p$-wave multipoles $P_{4}$ and $P_{5}$ and the longitudinal $s$-wave multipole $L_{0+}$ occur.

The five-fold differential cross section is given by

$$
\begin{aligned}
\frac{\mathrm{d} \sigma(\theta, \phi)}{\mathrm{d} \Omega^{\prime} d E^{\prime} d \Omega}=\Gamma( & \sigma_{T}+\epsilon \sigma_{L}+\epsilon \sigma_{T T} \cos 2 \phi \\
& +\sqrt{2 \epsilon(1+\epsilon)} \sigma_{T L} \cos \phi \\
& \left.+h \sqrt{2 \epsilon(1-\epsilon)} \sigma_{T L^{\prime}} \sin \phi\right)
\end{aligned}
$$

with the virtual photon flux $\Gamma$, the transverse photon polarization $\epsilon$ and the photon-proton center of mass angles $\theta$ and $\phi$ defined as in photoproduction with respect to the virtual photon direction.

The transverse cross section $\sigma_{T}$ and the longitudinal cross section $\sigma_{L}$ can be disentangled by varying $\epsilon$, for the other structure functions the differential cross section has to be measured over the corresponding angular range. The transverse-longitudinal interference $\sigma_{T L^{\prime}}$ can be extracted by a polarized electron beam with helicity $h$. 


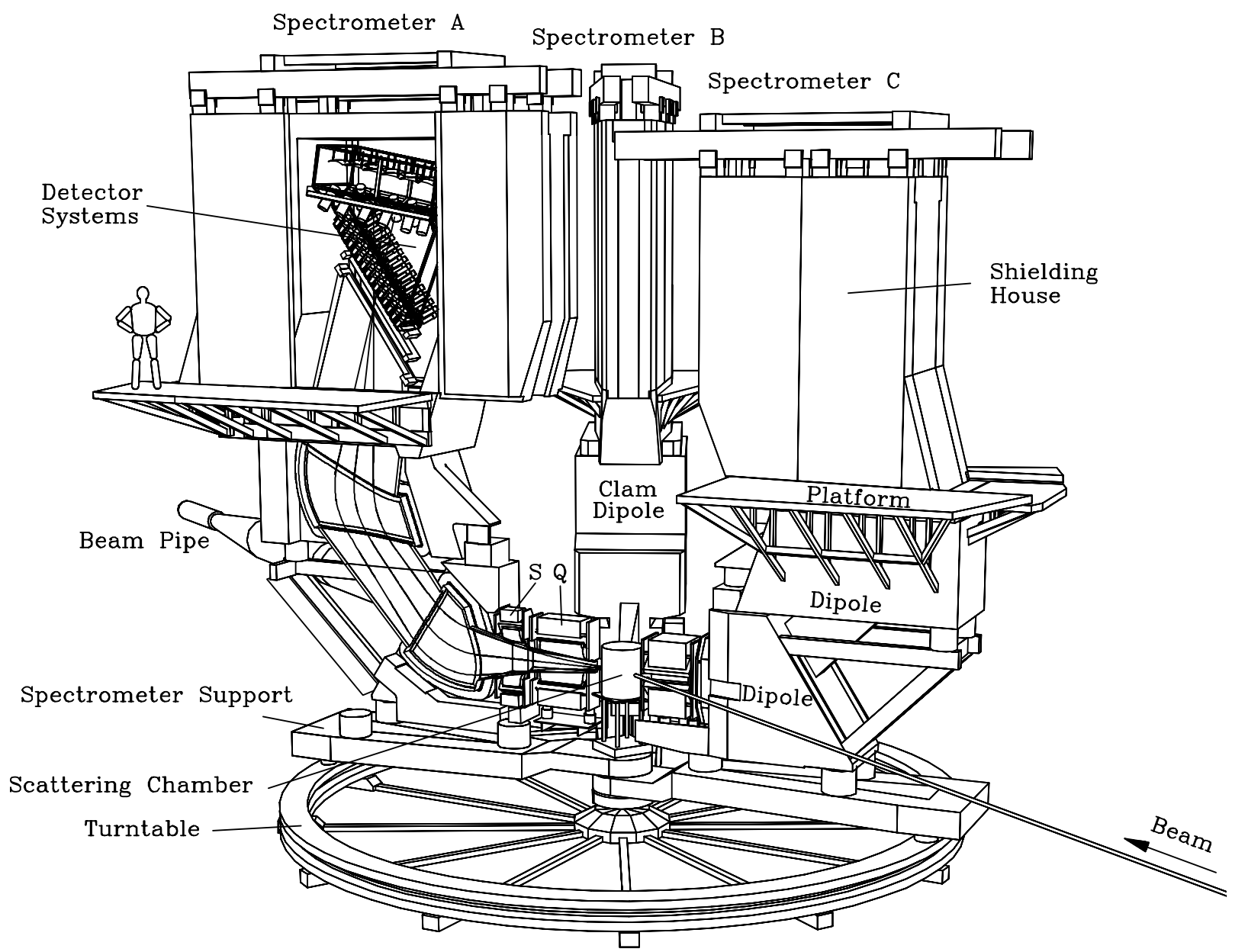

Fig. 6. The three-spectrometer setup of the A1 Collaboration at MAMI.

In the threshold region, the cross section can be further decomposed into $s$ - and $p$-wave multipoles:

$$
\begin{aligned}
\sigma_{T}(\theta) & =p / k_{\gamma}\left(A+B \cos \theta+C \cos ^{2} \theta\right), \\
\sigma_{L}(\theta) & =p / k_{\gamma}\left(A^{\prime}+B^{\prime} \cos \theta+C^{\prime} \cos ^{2} \theta\right), \\
\sigma_{T L}(\theta) & =p / k_{\gamma}(D \sin \theta+E \sin \theta \cos \theta), \\
\sigma_{T T}(\theta) & =p / k_{\gamma}\left(F \sin ^{2} \theta_{\pi}^{*}\right), \\
\sigma_{T L^{\prime}}(\theta) & =\sqrt{\frac{Q^{2}}{\omega^{* 2}}} p / k_{\gamma}(G \sin \theta+H \sin \theta \cos \theta),
\end{aligned}
$$

where $p / k_{\gamma}$ is the phase space ratio of pion CM momentum and photon CM equivalent momentum, and the angular coefficients are combinations of two $s$-wave and five $p$-wave multipoles:

$$
\begin{aligned}
& A=\left|E_{0+}\right|^{2}+\frac{1}{2}\left(\left|P_{2}\right|^{2}+\left|P_{3}\right|^{2}\right), \\
& B=2 \operatorname{Re}\left(E_{0+} P_{1}^{*}\right) \\
& C=\left|P_{1}\right|^{2}-\frac{1}{2}\left(\left|P_{2}\right|^{2}+\left|P_{3}\right|^{2}\right) \\
& D=-\operatorname{Re}\left(E_{0+} P_{5}^{*}+L_{0+} P_{2}^{*}\right) \\
& E=-\operatorname{Re}\left(P_{1} P_{5}^{*}+P_{4} P_{2}^{*}\right) \\
& F=\frac{1}{2}\left(\left|P_{2}\right|^{2}-\left|P_{3}\right|^{2}\right)
\end{aligned}
$$

$$
\begin{aligned}
A^{\prime} & =\left|S_{0+}\right|^{2}+\left|P_{5}\right|^{2}, \\
B^{\prime} & =2 \operatorname{Re}\left(S_{0+} P_{4}^{*}\right), \\
C^{\prime} & =\left(\left|P_{4}\right|^{2}-\left|P_{5}\right|^{2}\right), \\
G & =\operatorname{Im}\left(E_{0+}^{*} P_{5}+S_{0+}^{*} P_{2}\right), \\
H & =\operatorname{Im}\left(P_{1} P_{5}^{*}+P_{4} P_{2}^{*}\right) .
\end{aligned}
$$

\subsection{Unpolarized experiments}

A first electroproduction experiment at NIKHEF [10] estimated the $s$-wave cross section at threshold, a second experiment [11] tried to extract $p$-wave amplitudes in addition by measuring the pion emission angle in plane left and right from the virtual photon direction. While these pioneering works were in good agreement with ChPT, the predictive power was not full exploited, since the data has to be analyzed with assumptions from theory.

A more complete experiment was performed at MAMI [12] at the three-spectrometer setup of the A1 collaboration. In electro production, the recoil proton is detected in coincidence with the scattered electron. Very close to threshold, the boost by the Lorentz transformation from the center-of-mass system to the laboratory system focuses the full solid angle of the center of mass system into a narrow cone. 

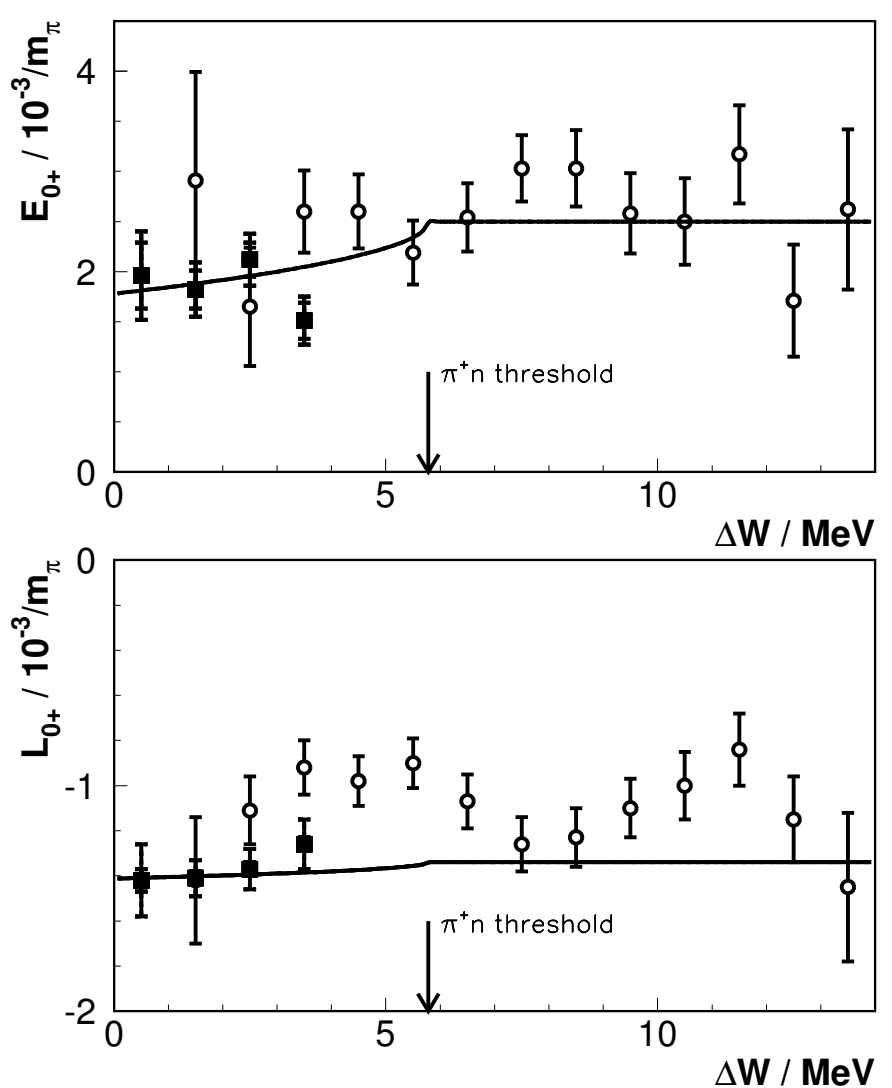

Fig. 7. The extracted $s$-wave multipoles of the MAMI data [12] (solid squares) in comparison with the NIKHEF data [11] (open circles) and a calculation in HBChPT [13] (line).

At MAMI, two high-resolution spectrometer of the A1 Collaboration were used for the detection of the scattered electron and the recoil proton (fig. 6). At a photon virtuality of $Q^{2}=0.1 \mathrm{GeV}^{2} / c^{2}$ up to a center-of-mass energy of $4 \mathrm{MeV}$ above threshold full coverage of the angular range was achieved. To separate transverse and longitudinal cross section, data were taken at three values of the photon polarization $\epsilon$.

For each bin in $\epsilon$ and energy the differential cross section was fitted to separate the structure functions $\sigma_{T}$ and $\sigma_{L}$ and the interference structure functions $\sigma_{T T}$ and $\sigma_{T L}$. Similar to photo production, the angular structure of the structure functions was fitted with the assumption of only $s$ - and $p$-waves contributing to the cross section.

Figure 7 shows the result for the $s$-wave multipoles in comparison with the results from NIKHEF and with the calculations in HBChPT [13].

As can be seen, the extracted multipoles are in agreement within the error bars and can be fitted by HBChPT. Actually, this result was surprising, since at that point a photon virtuality of $Q^{2}=0.1 \mathrm{GeV}^{2} / c^{2}$ was expected to be somewhat beyond the scope of HBChPT.

Therefore, a further experiment was performed at a lower virtuality of $Q^{2}=0.05 \mathrm{GeV}^{2} / c^{2}$ (ref. [14]). The same experimental technique was used as by the previous experiment, however the systematic errors are somewhat larger due to the lower outgoing proton momentum.
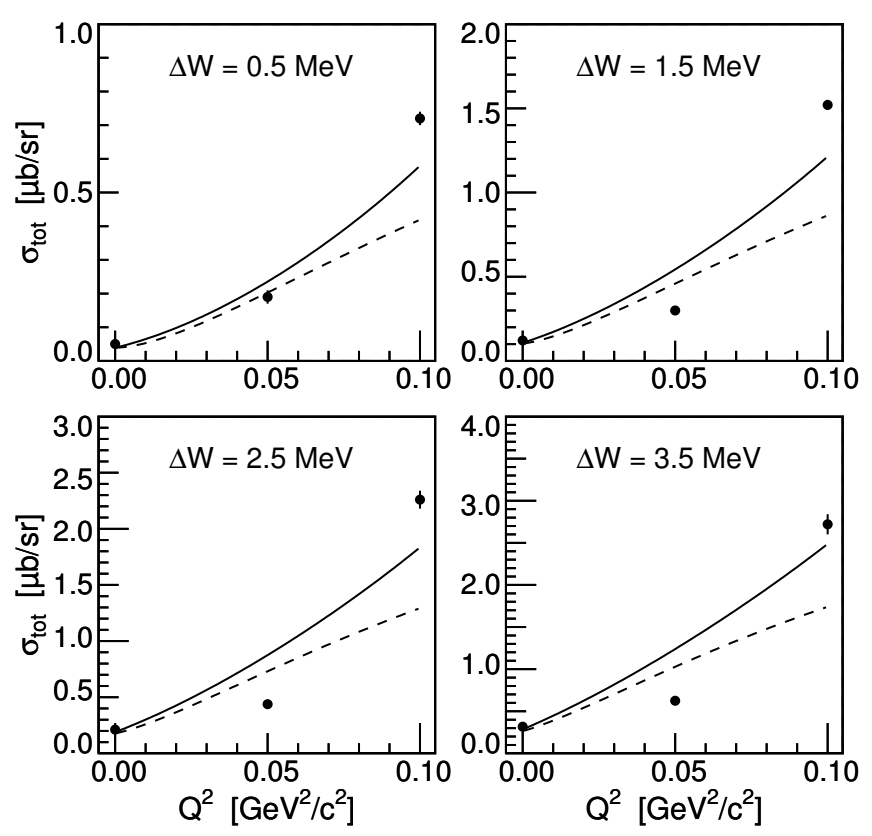

Fig. 8. The total cross section [14] versus photon virtuality (only MAMI data). The solid line shows the calculation in HBChPT [13].

Figure 8 summarizes the result of this experiment in comparison with the real photon data and the data at $Q^{2}=0.1 \mathrm{GeV}^{2} / c^{2}$. While the photon point and the higher $Q^{2}$ point was included in the fit of HBChPT, the middle $Q^{2}$ point was not. As can be seen, there was a clear discrepancy which has to be clarified.

\subsection{Extended momentum range}

The first step was, to extend the momentum range to be more sensitive to the interference structure functions. At MAMI an extended experiment was performed at a four-momentum transfer of $Q^{2}=0.05 \mathrm{GeV}^{2} / c^{2}$ [15]. Due to the extension of the momentum range to a centerof-mass energy of $40 \mathrm{MeV}$ above threshold the full angular range cannot be measured in a single kinematical setting. Several settings along the in plane angular range $\left(\phi=0^{\circ}, 180^{\circ}\right)$ are required to separate the differential cross section $\sigma_{0}=\sigma_{T}+\epsilon \sigma_{L}$ and the longitudinaltransverse interference structure function $\sigma_{L T}$.

To separate the transverse-transverse interference $\sigma_{T T}$, which has a dependence on the out-of-plane angle of $\cos 2 \phi$, the according out-of-plane acceptance is required. At MAMI, one of the high-resolution spectrometer (spectrometer $\mathrm{B}$ in fig. 6) can be tilted by up to $10^{\circ}$. By using this spectrometer as electron detector in extreme forward direction, this translates to $90^{\circ}$ in the center-of-mass frame.

Data were taken at 14 different kinematical settings and were combined to extract the structure functions. Figure 9 shows the structure functions versus the centerof-mass energy above threshold. For comparison, the calculation in HBChPT [13] was included (solid line). As 

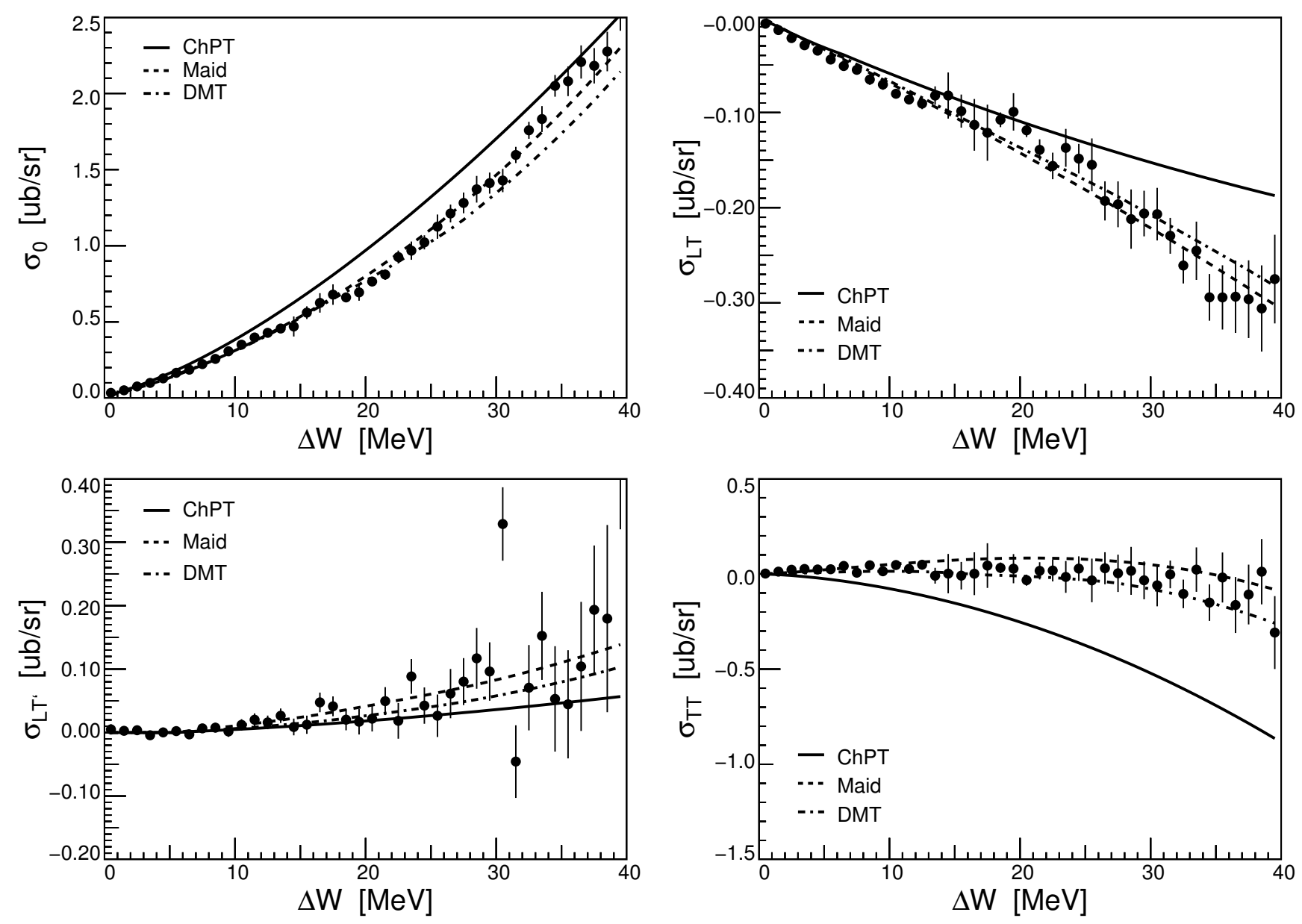

Fig. 9. Separated structure functions [15]. The solid line is the calculation in HBChPT [13], the dashed line the Maid model [16], and the dash-dotted line the DMT model [17].

stated before, this calculation overestimates the differential cross section. Even more striking, the derivation of the transverse-transverse interference is considerable, which is proportional to the difference of the $p$-wave multipoles $P_{2}$ and $P_{3}$, which are reproduced in good quality in photoproduction.

For comparison, the phenomenological model MAID [16] is included (dashed line). For the structure functions, this model is able to describe the data sets roughly. This model is basically a global fit to the existing data sets in photo- and electro-production and can be seen as check of the consistency of the data with the other existing data sets, however data at this photon virtuality and energy are scarth. The dynamical Dubna-MainzTaipeh model (DMT) [17] is shown as dashed-dotted line and is in good agreement with the data.

\subsection{Helicity asymmetry}

By using polarized electrons, the fifth structure function $\sigma_{L T^{\prime}}$ can be extracted. This structure function has the multipole contents $\sigma_{T L^{\prime}}\left(90^{\circ}\right) \propto \operatorname{Im}\left[L_{0+}^{*}\left(3 E_{1+}-M_{1+}+\right.\right.$ $\left.\left.M_{1-}\right)-E_{0+}\left(2 L_{1+}^{*}-L_{1-}^{*}\right)\right]$, i.e. it is basically propor- tional to the imaginary part of $L_{0+}$ multiplied by the large $\Delta(1232)$ multipole $M_{1+}$. Since it is very small, one has to define the helicity asymmetry

$$
\begin{aligned}
A_{T L^{\prime}}\left(\theta=90^{\circ}, \phi=-90^{\circ}\right) & =\frac{\sigma(h=1)-\sigma(h=-1)}{\sigma(h=1)+\sigma(h=-1)} \\
& =\frac{\sqrt{2 \epsilon(1-\epsilon)} \sigma_{T L^{\prime}}(\theta)}{\sigma_{T}(\theta)+\epsilon \sigma_{L}(\theta)-\epsilon \sigma_{T T}(\theta)}
\end{aligned}
$$

to reduce the systematic errors. The expected energy structure is given by the unitary cusp at the opening of the $\pi^{+}$threshold.

Figure 10 shows this asymmetry. Again, the calculations in HBChPT and the models MAID and DMT are included in the graph. This small asymmetry enhances the differences between the models, and as can be seen clearly, only the DMT model is able to describe the data.

\section{Coherent production from the deuteron}

The $\pi^{0}$ production on the neutron is in principle a strong prediction of HBChPT, since by the $\pi^{0}$ production from 


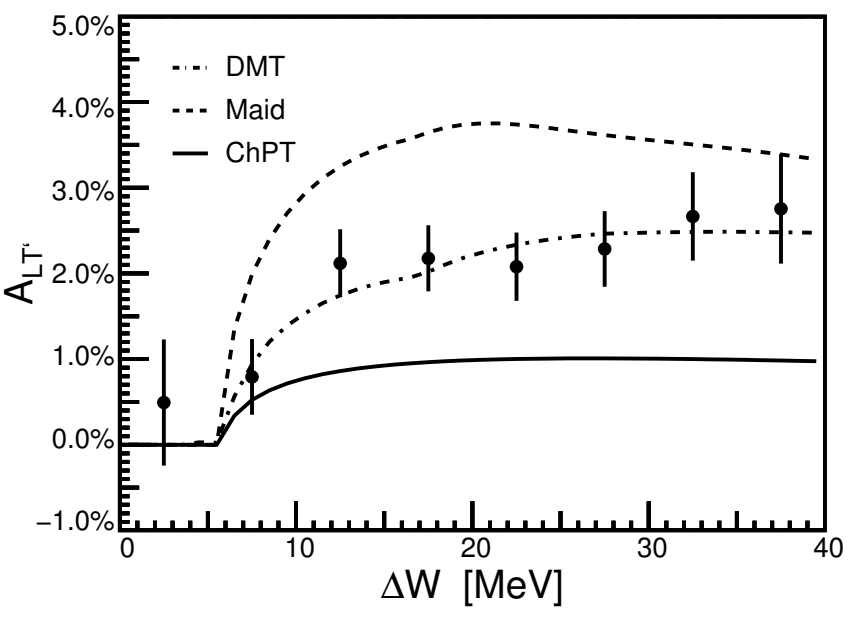

Fig. 10. The asymmetry $A_{L T^{\prime}}$ versus center-of-mass energy above threshold $\Delta W$. The solid line is the calculation in HBChPT [13], the dashed line the MAID model [16] and the dashed-dotted line the DMT model [17].

the proton nearly all low-energy constants are already fixed. Obvious, the lack of a free neutron target complicates the situation.

The use of the deuteron as neutron target requires detailed modeling of the deuteron structure as first step. Since the Fermi momenta of the nucleons in the deuteron is of the same order as the momenta of a threshold experiment, the coherent production is the most promising channel to get the deuteron structure and final state interaction under control.

By this choice, small cross sections are to be expected as pointed out first in ref. [18]. Due to the restrictions for the intermediate state of two neutrons in an $s$-wave due to Pauli blocking an exact cancelation between the $\pi^{+} n$ intermediate state in the $s$-wave for production from the proton and the rescattering graph by charge exchange on the spectator neutron occurs. This cancellation causes small $s$-wave cross sections on the one hand, but reduces the systematic uncertainties by the estimation of the final state interaction on the other hand.

\subsection{Coherent photoproduction}

A first photoproduction experiment was performed at SAL [19]. The decay photons of the pion were detected by the IGLOO detector, a large solid angle array of scintillators. By this technique, the coherent production from the deuteron cannot be separated from the break up channel with proton and neutron in the final state. The contribution of this channel was calculated in a model and subtracted from the data. The differential cross section was extrapolated to the threshold to extract the threshold $s$-wave amplitude.

A threshold value of $E_{d}=(-1.45 \pm 0.09) 10^{-3} / m_{\pi}$ was extracted. This value agreed within the error bar with a calculation in HBChPT [20], which gave a prediction for the coherent $s$-wave amplitude only.

\subsection{Coherent electroproduction}

In contrast to the technique of coherent photo production, in electro production the recoil deuteron is detected. This leads to clear and unique identification of the reaction channel by the missing mass of the pion. Without this subtraction, also the extraction of $p$-waves is possible.

While the theoretical advantage is obvious, the experimental challenge is large. In a threshold experiment at a four momentum transfer of $Q^{2}=0.1 \mathrm{GeV}^{2} / c^{2}$ the deuteron has a kinetic energy of $T \approx 30 \mathrm{MeV}$, which makes it difficult to achieve the necessary accuracy to identify the reaction.

At MAMI, the spectrometer setup is optimized to detect low energetic particles. By using a thin $\mathrm{LD}_{2}$ target with $3 \mathrm{~mm}$ path length in the deuterium and connecting the spectrometer vacuum system with the vacuum system of the target chamber, the multiple scattering and energy loss of the deuterons could be minimized. Still, the detection efficiency for deuterons is close to $80 \%$ (in comparison to $98 \%$ for protons) due to hadronic interactions of the deuteron.

Figure 11 shows the achieved missing mass resolution of the MAMI experiment [21]. Again, a full transverselongitudinal separation was performed by measuring a three different values of the photon polarization $\epsilon$ and the full center-of-mass angular range was covered up to a center-of-mass energy of $4 \mathrm{MeV}$ above threshold.

Since at that time only predictions for the $s$-wave amplitudes were available [22], a fit of the differential cross
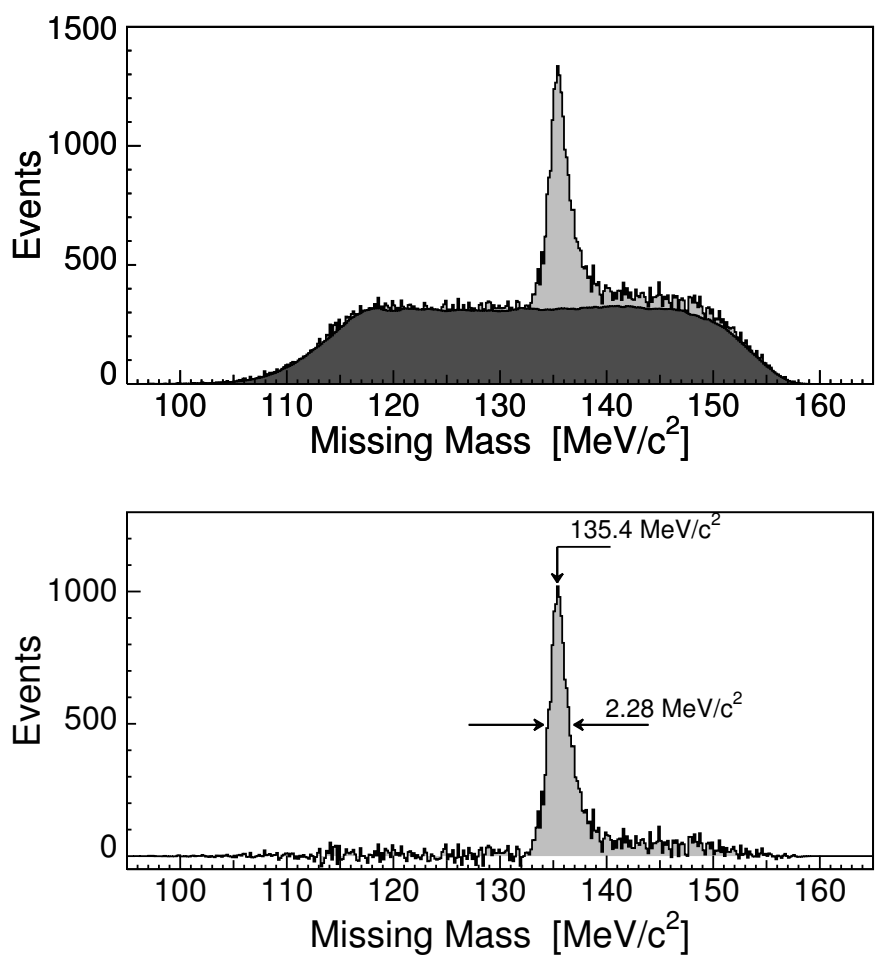

Fig. 11. Missing mass resolution for the reconstruction of the neutral pion [21]. 

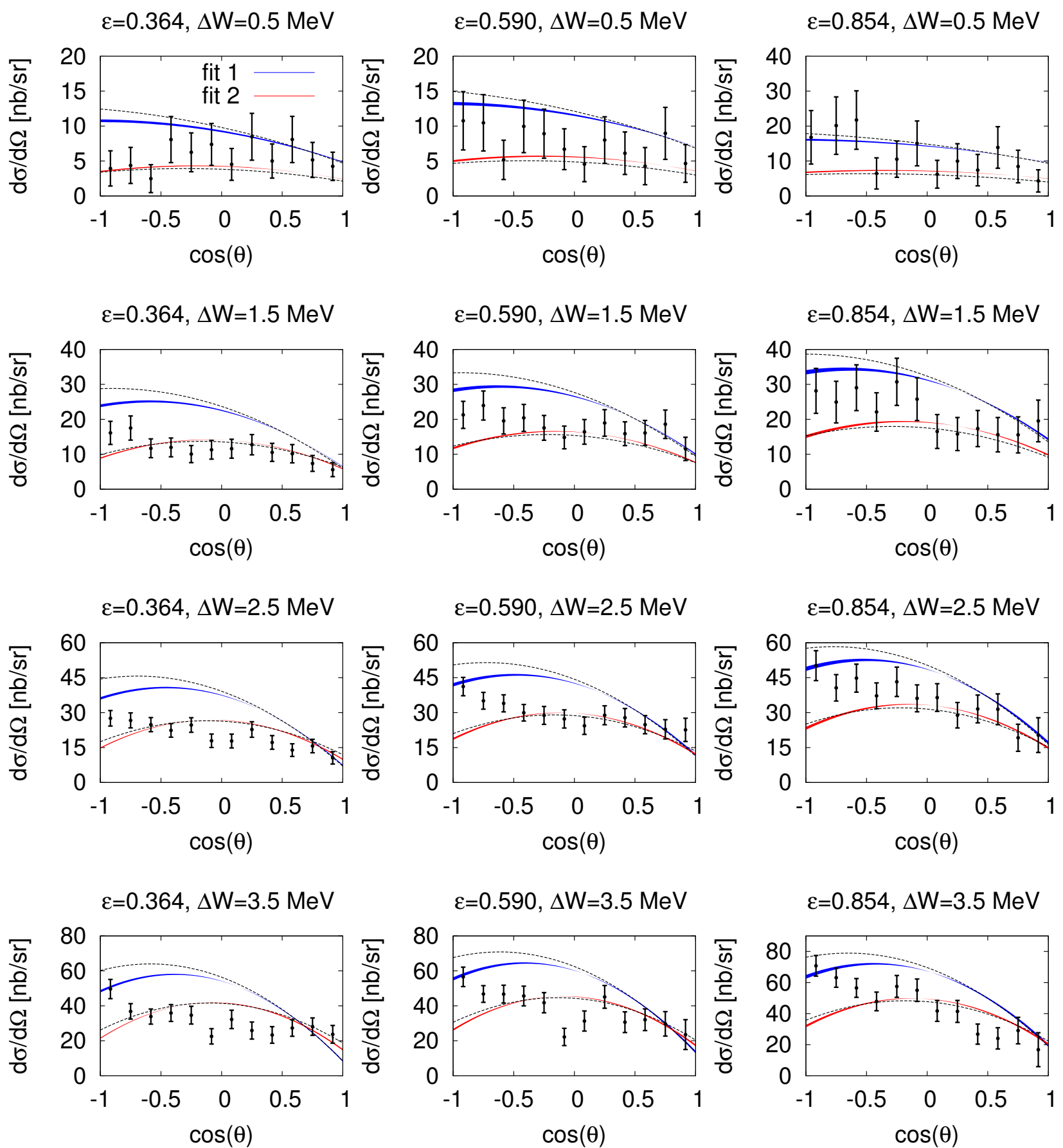

Fig. 12. The MAMI data on coherent electro production from deuteron [21] for three different values of the photon polarization $\epsilon$ in comparison with the calculations in HBChPT [23]. The upper curve shows the fit with fixed $L_{d}$, the lower curve shows the fit with two free low-energy constants. The dashed lines show the previous results from ref. [24].

section with the usual assumptions of $s$ - and $p$-waves with the known energy dependence was performed to extract the threshold $s$-wave amplitudes. It has to be stressed, that this fit ignores completely the deuteron structure and final state interaction, leading to a large systematic errors in the extracted amplitudes. Since $L_{d}$ is very large, for $E_{d}$ only an upper limit could be extracted. The amplitudes were extracted to

$$
\begin{aligned}
& \left|E_{d}\right| \leq 0.42 \cdot 10^{-3} / m_{\pi}, \\
& \left|L_{d}\right|=(0.50 \pm 0.11) \cdot 10^{-3} / m_{\pi} .
\end{aligned}
$$

The transverse multipole $E_{d}$ was within agreement with the calculations as at the photon point, while the 
calculations overestimated the longitudinal multipole by a factor of 2 .

The situation improved a lot by a new calculation by the same group [23], which now used a complete multipole decomposition and extracted $s$ - and $p$-waves. By this, the comparison has not to be done on the level of the questionable extracted $s$-wave amplitudes, but can be done directly with the differential cross section.

Figure 12 shows the result of their calculation in comparison with the MAMI data. The plot shows two kind of fits: the first was performed by fixing the $L_{d}$ multipole to the extracted value of the data set, reducing the number of parameters to a single low-energy constant. This fit, however, does not describe the data set completely. The second fit was performed with two free parameters and describes the data.

\section{Summary and outlook}

While the data basis on pion threshold production is now quite large, there are still a number of unsolved problems. The strong $Q^{2}$-dependence of the total cross section (fig. 8) seems to be unlikely and might indicate the large systematic errors of the data sets as quoted by the authors.

To resolve this puzzle, a dedicated experiment was performed by MAMI, where three different $Q^{2}$ values were measured within one experiment and with emphasis on reducing the systematic error [25]. This experiment is under analysis, but preliminary data seems to indicate, that the $Q^{2}=0.1 \mathrm{GeV}^{2} / c^{2}$ data set is somewhat high. Since that data set is included in the fit of HBChPT, a refit of the theory seems to be necessary as soon as the new data set is published.

All cited calculations are based on the heavy-baryon formalism. Meanwhile, better regularization schemes are on the market and an improvement of the calculations can be expected. Especially in electroproduction, where the $p$ waves are not yet calculated to the same order as in photo production, a considerable improvement is possible.
The success of ChPT in the $S U(2)$ sector encourages one to extend this program to the strangeness threshold. At MAMI a new kaon spectrometer is under construction, and kaon threshold production will be among the first experiments with this spectrometer.

\section{References}

1. E. Mazzucato et al., Phys. Rev. Lett. 57, 3144 (1986).

2. R. Beck et al., Phys. Rev. Lett. 65, 1841 (1990).

3. A. Schmidt et al., Phys. Rev. Lett. 87, 232501 (2001).

4. P. de Baenst, Nucl. Phys. B 24, 633 (1970); I.A. Vainshtein, V.I. Zakharov, Nucl. Phys. B 36, 589 (1972).

5. V. Bernard, N. Kaiser, J. Gasser, U.-G. Meißner, Phys. Lett. B 268, 291 (1991); V. Bernard, N. Kaiser, U.-G. Meißner, Z. Phys. C 70, 483 (1996).

6. J.C. Bergstrom et al., Phys. Rev. C 53, R1052 (1996).

7. M. Fuchs et al., Phys. Lett. B 368, 20 (1996); A.M. Bernstein et al., Phys. Rev. C 55, 1509 (1997).

8. V. Bernard et al., Eur. Phys. J. A 11, 209 (2001).

9. O. Hanstein et al., Phys. Lett. B 399, 13 (1997).

10. T.P. Welch et al., Phys. Rev. Lett. 69, 2761 (1992).

11. H.B. van den Brink et al., Phys. Rev. Lett. 74, 3561 (1995).

12. M.O. Distler et al., Phys. Rev. Lett. 80, 2294 (1998).

13. V. Bernard, N. Kaiser, U.-G. Meißner, Nucl. Phys. A 607, 379 (1996); 633, 695 (1998)(E).

14. H. Merkel et al., Phys. Rev. Lett. 88, 012301 (2002).

15. M. Weis, Doctorate Thesis, Mainz, 2003.

16. D. Drechsel et al., Nucl. Phys. A 645, 145 (1999); S.S. Kamalov et al., Phys. Lett. B 522, 27 (2001).

17. S.S. Kamalov et al., Phys. Rev. Lett. 83, 4494 (1999); Phys. Rev. C 64, 032201 (2001).

18. M. Rekalo, E. Tomasi-Gustafsson, Phys. Rev. C 66, 015203 (2002).

19. J.C. Bergstrom et al., Phys. Rev. C 57, 3203 (1998).

20. S.R. Beane et al., Nucl. Phys. A 618, 381 (1997).

21. I. Ewald et al., Phys. Lett. B 499, 238-244 (2001).

22. V. Bernard, H. Krebs, U.-G. Meißner, Phys. Rev. C 61, 58201 (2000).

23. H. Krebs, V. Bernard, U.-G. Meißner, Eur. Phys. J. A 22, 503-514 (2004).

24. H. Krebs, V. Bernard, U.-G. Meißner, Nucl. Phys. A 713, 405 (2003).

25. J. García Llongo, Diploma Thesis, Mainz, in preparation. 